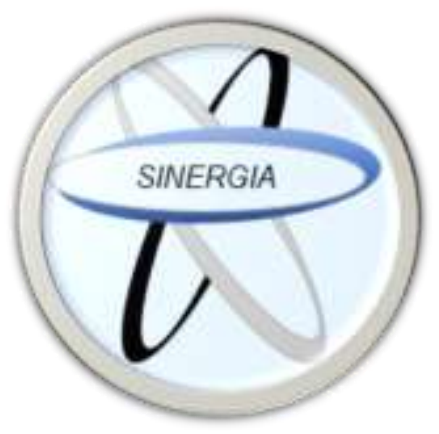

Volumen 4 Número 8

PUBLICACION MENSUAL

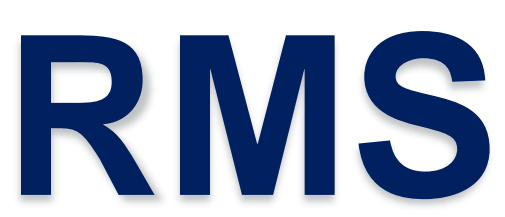

Revista Médica Sinergia

Agosto 2019

https://doi.org/10.31434/rms.v4i8.264

Artículo de investigación:

\title{
Correlación entre marcadores serológicos y ecográficos en pacientes con hígado graso no alcohólico y diabetes mellitus tipo 2
}

Correlation between serological and echographic markers to patients with non-alcoholic fatty liver and diabetes mellitus type 2

Autores:

Dra. Naibel Quevedo Ramírez

Dr. Raydel Pérez Castillo

Dra. Isnielly Virgen Sánchez Gendriz
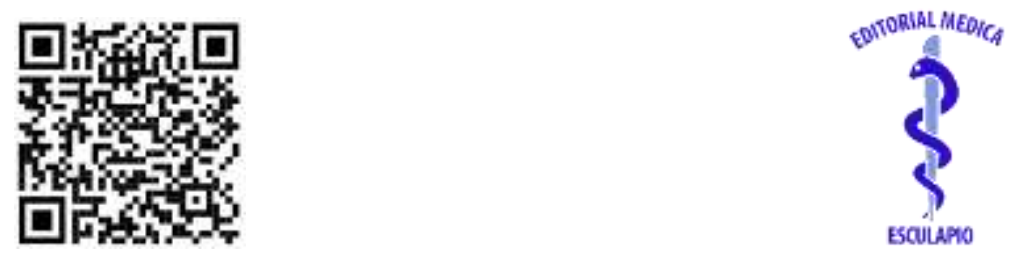


\section{CUERPO EDITORIAL}

\section{DIRECTORA}

- Dra. Margarita Karol Malpartida Ampudia, Médico independiente, San José, Costa Rica.

\section{EDITOR}

- Dr. Esteban Sánchez Gaitán, Caja costarricense del Seguro Social, Limón, Costa Rica.

\section{CONSEJO EDITORIAL}

- Dr. Cesar Vallejos Pasache, Hospital III lquitos, Loreto, Perú.

- Dra. Anais López, Hospital Nacional Edgardo Rebagliati Martins, Lima, Perú.

- Dra. Ingrid Ballesteros Ordoñez, Pontificia Universidad Javeriana, Bogotá, Colombia.

- Dra. Mariela Burga, Hospital Nacional Edgardo Rebagliati Martins. Lima, Perú.

- Dra. Patricia Santos Carlín, Ministerio de Salud (MINSA). Lima, Perú.

- Dr. Raydel Pérez Castillo, Centro Provincial de Medicina Deportiva Las Tunas, Cuba.

\section{COMITÉ CIENTÍFICO}

- Dr. Zulema Berrios Fuentes, Ministerio de Salud (MINSA), Lima, Perú.

- Dr. Gerardo Francisco Javier Rivera Silva, Universidad de Monterrey, Nuevo León, México.

- Dr. Gilberto Malpartida Toribio, Hospital de la Solidaridad, Lima, Perú.

- Dra. Marcela Fernández Brenes, Caja costarricense del Seguro Social, Limón, Costa Rica

- Dr. Hans Reyes Garay, Eastern Maine Medical Center, Maine, United States.

- Dr. Steven Acevedo Naranjo, Saint- Luc Hospital, Quebec, Canadá.

- Dr. Luis Osvaldo Farington Reyes, Hospital regional universitario Jose Maria Cabral y Baez, Republica Dominicana.

- Dra. Caridad Maria Tamayo Reus, Hospital Pediátrico Sur Antonio María Béguez César de Santiago de Cuba, Cuba.

- Dr. Luis Malpartida Toribio, Hospital Nacional Daniel Alcides Carrión, Callao, Perú.

- Dra. Allison Viviana Segura Cotrino, Médico Jurídico en Prestadora de Salud, Colombia.

\section{EQUÍPO TÉCNICO}

- Msc. Meylin Yamile Fernández Reyes, Universidad de Valencia, España.

- Lic. Margarita Ampudia Matos, Hospital de Emergencias Grau, Lima, Perú.

- Ing. Jorge Malpartida Toribio, Telefónica del Perú, Lima, Perú.

- Srta. Maricielo Ampudia Gutiérrez, George Mason University, Virginia, Estados Unidos.

\section{EDITORIAL ESCULAPIO}

50 metros norte de UCIMED, Sabana Sur, San José-Costa Rica Teléfono: 8668002

E-mail:

revistamedicasinergia@gmail.com

\section{ENTIDAD EDITORA}

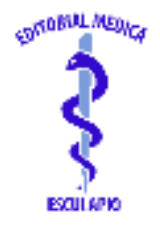

Rica

\section{SOMEA}

SOCIEDAD DE MEDICOS DE AMERICA

Frente de la parada de buses Guácimo, Limón. Costa

Teléfono: 8668002

Sociedaddemedicosdeamerica@hotmail.com

https://somea.businesscatalyst.com/informacion.html 


\section{Correlación entre marcadores serológicos y ecográficos en pacientes con hígado graso no alcohólico y diabetes mellitus tipo 2}

Correlation between serological and echographic markers to patients with nonalcoholic fatty liver and diabetes mellitus type 2

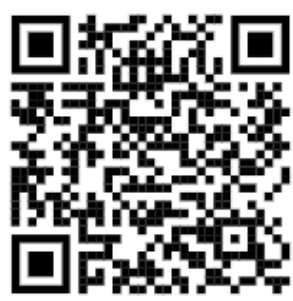

1 Médica especialista en Medicina Familiar e Imagenología en el Hospital Salvador Allende, La Habana, Cuba. naibelar@nauta.cu

2

Médico especialista en $K$ instructor, Centro Provincial de Medicina del Deporte, Las Tunas, Cuba. raydelperezc@nauta.cu

${ }^{3}$ Médica especialista en Imagenología, Hospital Hermanos Ameijeiras, Cuba. ivirgen@nauta.cu
${ }^{1}$ Dra. Naibel Quevedo Ramírez Hospital Salvador Allende, La Habana, Cuba

(D) https://orcid.org/0000-0002-5769-2314

${ }^{2}$ Dr. Raydel Pérez Castillo Centro Provincial de Medicina del Deporte, Las Tunas, Cuba (D) https://orcid.org/0000-0001-9454-5375

${ }^{3}$ Dra. Isnielly Virgen Sánchez Gendriz Hospital Hermanos Ameijeiras, La Habana, Cuba

(D) https://orcid.org/0000-0003-2703-0659

RECIBIDO

CORREGIDO

ACEPTADO

$10 / 06 / 2019$

$12 / 07 / 2019$

$28 / 07 / 2019$

\section{RESUMEN}

Introducción: Los diabéticos tipo 2 tienen un alto riesgo de padecer hígado graso no alcohólico. La detección e intervención temprana podrían reducir el riesgo cardiovascular, prevenir sus complicaciones y reducir su progresión. El objetivo es evaluar la correlación entre los marcadores por ecografía abdominal y marcadores serológicos indirectos en pacientes diabéticos tipo 2 con hígado graso no alcohólico.

Método: Se realizó un estudio descriptivo, correlacional y transversal. Se estudiaron 33 pacientes por ecografía abdominal para evaluar la severidad de la esteatosis hepática. Los marcadores serológicos indirectos fueron glicemia, colesterol total, triglicéridos, alanino-transaminasa, aspartatotransaminasa y ganmaglutamil-transferasa. Se contrastaron los resultados de ambos marcadores mediante $T$ de Student, prueba de ajuste de Kolmogorov y análisis de varianza.

Resultados: La edad promedio fue de 54,9 $\pm 9,1$ años. Se observó con más frecuencia marcador hiperecogénico moderado (45,5\%) con patrón difuso. Los marcadores serológicos indirectos más alterados fueron glicemia ( $n=29 ; 87,8 \%)$, triglicéridos ( $n=25 ; 75,8 \%)$, colesterol total $(n=22$; $66,7)$ y alanino-transaminasa $(n=21 ; 63,6 \%)$. Los niveles de glicemia y enzima ASAT ofrecieron valores de Rho Spearman de $r=0,65$ y $r=0,61$ 
respectivamente.

Conclusión: El desarrollo del hígado graso no alcohólico pareciera estar ligado a descompensación metabólica crónica e insulinorresistencia. Los niveles de glicemia y enzima ASAT fueron los marcadores serológicos indirectos que presentaron la mayor correlación estadística con los grados de lesión hepática diagnosticada por ecografía abdominal. Se recomienda el uso de modelos predictivos a partir de variables clínicas, marcadores serológicos indirectos y ecográficos en el diagnóstico y seguimiento a pacientes con hígado graso no alcohólico por su sencillez, y buena correlación con técnicas más complejas. La estratificación de riesgo con el empleo de dichos herramientas posibilita implementar medidas tempranas para reducir la morbimortalidad por causa cardiovascular.

PALABRAS CLAVE: enfermedad del hígado graso no alcohólico; hígado graso; diabetes mellitus tipo 2; ultrasonido.

\section{ABSTRACT}

Introduction: Type 2 diabetics have a high risk of suffering from fatty liver disease alcoholic. Detection and early intervention may reduce cardiovascular risk, prevent their complications and reduce their progression. The objective is to evaluate the correlation between the markers by abdominal ultrasound and indirect serological markers in type 2 diabetic patients with non-alcoholic fatty liver.

Method: A descriptive, correlational and transversal study was carried out. Thirty three patients were studied to evaluate the severity of hepatic steatosis by abdominal ultrasound. The indirect serological markers were glycemia, total cholesterol, triglycerides, alanine transaminase, aspartate transaminase and ganmaglutamyl transferase. The results of both markers by Student's T test, Kolmogorov adjustment test and variance analysis.

Results: The average age was $54.9 \pm 9.1$ years. Moderate hyperechogenic marker $(45.5 \%)$ with diffuse pattern was more frequently observed. The most altered indirect serological markers were glycemia $(n=29,87.8 \%)$, triglycerides $(n=25,75.8 \%)$, total cholesterol $(n=22,66.7)$ and alaninetransaminase $(n=21) .63 .6 \%)$. The levels of glycemia and enzyme ASAT offered Rho Spearman values of $r=0.65$ and $r=0.61$ respectively.

Conclusion: The development of nonalcoholic fatty liver appears to be linked to chronic metabolic decompensation and insulin resistance. The levels of glycemia and ASAT enzyme were the indirect serological markers that had the highest statistical correlation with the degrees of liver injury diagnosed by abdominal ultrasound. It is recommended the use of predictive models from clinical variables, Indirect and ultrasound serological markers in the diagnosis and follow-up of patients with non-alcoholic fatty liver for its simplicity, and good correlation with more techniques complex. Risk stratification with the use of these tools enables implement early measures to reduce morbidity and mortality due to cardiovascular causes.

KEYWORDS: non-alcoholic fatty liver disease; fatty liver; diabetes mellitus type 2; ultrasonics. 


\section{INTRODUCCIÓN}

Los diabéticos tipo 2 tienen un alto riesgo de padecer hígado graso no alcohólico (EHGNA) en un 50 a $70 \%$. Hoy en día, se considera que la EHGNA es la manifestación hepática del síndrome metabólico (1). Por tanto, es importante la detección e intervención temprana para reducir el riesgo cardiovascular, prevenir las posibles complicaciones $(2,3,4)$. El escenario clínico-patológico es similar al observado en la lesión hepática inducida por alcohol; sin embargo, ocurre en sujetos sin antecedentes de uso o abuso de alcohol (5,6). A muchos pacientes se les diagnostica la EHGNA tras un hallazgo accidental por una mayor concentración de enzimas hepáticas (7).

La prevalencia del EHGNA ha pasado a ser la causa más frecuente de enfermedad crónica hepática al aumentar hasta el $75.1 \%$ desde el $2008(8,9,10)$. Se estima que en los próximos años una de las principales causas de cirrosis y de necesidad de trasplante de hígado será la enfermedad por EHGNA (1). Para el 2018 según el Anuario Estadístico de Salud en Cuba; de cada 1000 personas 64,3 padecen diabetes mellitus; por su parte, la mortalidad por enfermedades hepáticas ha trascendido a 21,1 por cada 100000 habitantes, reportándose un exceso en los decesos por diabetes mellitus en el sexo femenino. Otras consideraciones en este informe refieren que la tasa de años de vida potencial perdidos se eleva por cirrosis u otras enfermedades crónicas del hígado (11). Otras investigaciones reportan una alta incidencia de obesidad en la población adulta $(42,4 \%)$ y un $26 \%$ de personas con EHGNA $(12,13)$.
La importancia clínica del EHGNA y las limitaciones de la biopsia, han intensificado la necesidad de buscar estrategias diagnósticas no invasivas. El impacto real estaría en herramientas que permitan una integración clínica, epidemiológica e imagenológica en el diagnóstico precoz y el seguimiento para la reducción de la mortalidad cardiovascular asociada. Ante esta justificación el objetivo del presente estudio es evaluar la correlación entre los marcadores por ecografía abdominal y marcadores serológicos indirectos en pacientes diabéticos tipo 2 con hígado graso no alcohólico.

\section{MÉTODO}

Se realizó un estudio descriptivo, correlacional y transversal, para evaluar la correlación entre los marcadores por ecografía abdominal y marcadores serológicos indirectos, en pacientes diabéticos tipo 2 con EHGNA. La población estuvo conformada (231 casos) por todos pacientes atendidos en la consulta protocolizada de Diabetes Mellitus Complicada, del Hospital ClínicoQuirúrgico "Hermanos Ameijeiras" en La Habana, Cuba; desde enero de 2016 a agosto de 2018. El muestreo fue no probabilístico intencional, quedando conformado por 33 pacientes según las recomendaciones de la guía para el manejo de EHGNA de las Asociaciones Europea para el Estudio del Hígado, Diabetes y Obesidad (14) y la American Asociation for Study Liver Diseases (15). Los criterios establecidos fueron:

Inclusión: Edad superior a los 18 años, antecedentes de diabéticos mellitus tipo 2, y diagnóstico de hígado graso no 
alcohólico y aceptación de consentimiento informado.

Exclusión: Casos con estudios serológicas positivos a virus de Hepatitis $B$ y $\mathrm{C}$, antecedentes de abuso de alcohol (14 bebidas alcohólicas estándar por semana en mujeres y 21 en hombres), diagnóstico de ecogenicidad hepática Normal (Grado 1), antecedentes de otras enfermedades hepáticas (hemocromatosis, déficit de alfa 1 antitripsina, enfermedad de Wilson, hepatitis autoinmune, cirrosis biliar primaria y colangitis esclerosante).

Se analizaron las variables: edad, sexo, glicemia (normal: $3,3-5,5$ y alterado $\geq 5,6$ $\mathrm{mmol} / \mathrm{L}$ ), colesterol total (normal: $\leq 5,2$ y alterado: $\geq 5,3 \mathrm{mmol} / \mathrm{L})$, triglicéridos (normal: $\leq 1,8$ y alterado $\geq 1,9 \mathrm{mmol} / \mathrm{L}$ ). Se consideraron las siguientes determinaciones enzimáticas: Alaninotransaminasa (ALAT): normal $\leq 39$ y alterado $\geq 40 \quad \mathrm{U} / \mathrm{L} ; \quad$ Aspartatotransaminasa (ASAT) normal: normal $\leq$ 39 y alterado $\geq 40 \mathrm{U} / \mathrm{L}$; Ganmaglutamiltransferasa (GGT) normal: $\leq 32$ en mujeres y 45 en los hombres y alterado $\geq$ 33 ó $46 \mathrm{U} / \mathrm{L}$ respectivamente. Estos parámetros humorales se analizaron en el laboratorio clínico del Hospital "Hermanos Ameijeiras" usando los métodos de la química clínica Cobas 500 (Diagnóstico de Roche, Alemania) según el soporte técnico del fabricante.

Se utilizó un equipo ecográfico marca Philip, con transductores de 3.5 y $5 \mathrm{MHz}$. Las imágenes se visualizaron en 6 cortes preestablecidos según la literatura internacional sin cambio de ganancia, realizados por un mismo explorador, precisando las siguientes características:

1. Ecotextura: la esteatosis se observa como un incremento de la ecogenicidad hepática con ecos muy finos y condensados, con apariencia de un "hígado brillante". Resulta más evidente en comparación con la ecogenicidad del parénquima renal o esplénico.

2. Atenuación sonora: Aparece tras el súbito descenso en la transmisión ecográfica, se establece a los 405 $\mathrm{cm}$ de profundidad, haciendo a las estructuras más recónditas difíciles de visualizar, sobre todo en los segmentos posteriores del hígado.

3. Heterogeneidad:Hígado uniformemente heterogéneo, con irregularidad del contorno capsular.

4. Visibilidad de los bordes: El hígado ocupa el campo completo sin bordes visibles.

5. Visibilidad vascular: Se evalúa la visualización de las paredes de las estructuras vasculares intrahepáticas (venas porta y hepáticas) y de la vesícula biliar.

6. Borramiento: Puede ser parcial o con pérdida de la definición del diafragma.

Basado en los anteriores criterios se determinó la presencia de aumento de la ecogenicidad hepática, y gradación según criterios validados por varios autores $(16,17,18,19)$.

Grado 1: Normal

Grado 2: Leve. Cuando se observa un aumento ligero de la ecogenicidad del parénquima hepático respecto a la cortical renal, con escasa distorsión de su ecoestructura (grano fino), con clara definición de las paredes de las venas porta y hepáticas, así como visualización del diafragma, y no hay atenuación del sonido.

Grado 3: Moderada. Caracterizada por un aumento moderado de la ecogenicidad hepática con respecto a la 
cortical renal y al parénquima del bazo, y borramiento parcial de las paredes de las venas porta y hepáticas, y dificultad para la valoración del diafragma; y hay atenuación del sonido.

Grado 4: Severa. El hígado es intensamente ecogénico, visualización de las paredes de los vasos intrahepáticos y del diafragma escasa o nula; y hay atenuación del sonido.

Como fuente de información se utilizaron las historias clínicas individuales, los informes del ecografía abdominal y laboratorio clínico. Se confeccionó un modelo de registro que permitió la recolección de datos, con el objetivo de recoger los variables $\mathrm{e}$ indicadores relacionados con la enfermedad de base. La información recogida se procesó con ayuda del paquete estadístico Infostat/L. Se utilizaron medidas univariadas de estadística descriptiva para el resumen de la información (tendencia central y dispersión) y bivariadas para la demostración de correlación entre los grados de lesión hepática y las alteraciones laboratorios (Prueba de ajuste de Kolmogorov, T Student, análisis de varianza (ANAVA) y coeficiente de Rho de Spearman. Los resultados se presentaron en tablas de entradas múltiples, gráfica de dispersión, caja (Box-Plot) e histograma con el fin de darle salida al propósito planteado.

El estudio se realizó en correspondencia a las normas éticas de Helsinki. El grupo de investigadores protegió a los participantes de cualquier molestia física - mental que pudiera ocasionar la investigación, aun cuando esta representaba un beneficio potencial para ellos. La información relacionada con la identidad de los enfermos se trató de forma confidencial, y fue manejada sólo por el personal especializado que participó en la investigación. A los sujetos participantes se les dio una explicación detallada sobre la investigación, su importancia, así como en qué consistía la exploración clínica.

Los pacientes recibieron la información necesaria para decidir su participación en la investigación. Entre otros aspectos se explicaron el objetivo del estudio, los beneficios esperados y el resto de los elementos que permitieron al individuo valorar su aceptación o no de participar en el mismo, con un carácter voluntario y la posibilidad de abandonar el mismo cuando así lo deseara, sin repercusión o enjuiciamiento alguno. Se contó con el consentimiento escrito, obtenido a través de la firma del modelo de Consentimiento Informado. Ninguno de los resultados aquí planteados se considera experimental, la obtención del mismo parte de procedimientos médicos validados y con aprobación legal.

\section{MARCO TEORICO}

EI EHGNA fue descrito originalmente en un grupo de enfermos obesos con esteatosis hepática en el decenio de 1950. En 1980, Ludwig et al, en la Clínica Mayo, describieron los casos de 20 pacientes obesos, diabéticos y no alcohólicos que mostraban en la biopsia hepática signos similares a los de personas con hepatopatía alcohólica, razón por la que se introdujo el término esteatohepatitis no alcohólica (7). El hígado graso es una afección que se caracteriza por la acumulación de ácidos grasos libres y triglicéridos en el citoplasma de los hepatocitos. Esta condición es debida a un desequilibrio en cualquiera de las sendas involucradas en 
la entrega, la síntesis, exportación u oxidación de triglicéridos (16).

Para definir la enfermedad como EHGNA se requiere que haya una evidencia del 5 $\%$ de esteatosis hepática, ya sea por métodos imagenológicos o histológico; y que no existan causas secundarias para la acumulación de grasa hepática, con o sin inflamación, degeneración balonoide del hepatocito y/o fibrosis, excluyendo causas secundarias de acumulación grasa como consumo excesivo de alcohol, el uso de medicamentos esteatogénicos o trastornos hereditarios del metabolismo según la American Asociation for Study Liver Diseases $(1,15,20)$.

La enfermedad hepática grasa no alcohólica comprende rangos que oscilan entre la esteatosis simple y la esteatohepatitis no alcohólica (EHNA), afección que puede ocasionar diversos grados de fibrosis en el hígado, e incluso puede progresar a la cirrosis hepática o hepatocarcinoma (21). La EHGNA se caracteriza por la presencia de esteatosis hepática sin la evidencia de lesión hepatocelular, a diferencia de la esteatohepatitis no alcohólica que se define como la presencia de esteatosis hepática e inflamación, y lesión del hepatocito, con o sin fibrosis (22).

\section{EPIDEMIOLOGÍA}

Las enfermedades crónicas del hígado tienen una alta morbimortalidad a nivel mundial, la prevalencia e incidencia son variables dependiendo de la población estudiada. Los datos diagnósticos son diversos a causa las técnicas y criterios utilizados (23); se estima entre 6,3 y 33 $\%$ (media $25 \%$ en la población general lo padece, basado en una valoración de estudios imagenológicos. Por otro lado, la prevalencia estimada de esteatohepatitis no alcohólica es más baja (3 a $5 \%$ ), mientras que la de cirrosis secundaria a EHNA, no ha sido muy reportada $(1,18,24)$.

Según datos de la literatura científica entre el 20 a $30 \%$ de la población europea tiene EHGNA, con un pico de incidencia en el Reino Unido de 39 por cada 100000 habitantes. En África existen pocos estudios epidemiológicos precisos, con excepción de una investigación donde se reporta que cerca del $10 \%$ de la población Nigeriana presentan distintos grados de esteatosis hepática $(18,25)$. Estudios en japoneses informan por su parte una incidencia entre 31 y 86 casos de EHGNA por cada 1000 personas por año; sin embargo, en Inglaterra la tendencia es mucho más baja (29 casos por 100000 persona por año) (26).

Otras fuentes citan que los países industrializados presentan una incidencia entre 40 y $50 \%$ de la población general, con tasas más elevada de EHGNA en diabéticos tipo 2. En los Estados Unidos de América el predominio se estima, aproximadamente entre 3 a $26 \%$, con tasas más alta en poblaciones de alto riesgo. En la población americana en general 30,1 millones de adultos son obesos con esteatosis hepática y cerca de 8,6 millones tienen esteatohepatitis comprobada por biopsia (9).

Las comorbilidades metabólicas asociadas con el diagnóstico de EHGNA incluyen obesidad (51.34\%), diabetes mellitus tipo 2 (22.51\%), hiperlipidemia (69.16\%), hipertensión arterial (39.34\%) y Set $(42.54 \%)$. Se ha reportado que más del $76 \%$ de los pacientes con DM2 tienen 
EHGNA, y más del $90 \%$ de los pacientes con obesidad mórbida sometidos a cirugía bariátrica $(1,8)$.

\section{FISIOPATOGENIA E HISTORIA NATURAL}

El primer cambio en la EHGNA es una acumulación de lípidos (en especial triglicéridos) en los hepatocitos. El estrés oxidativo resultante de la oxidación mitocondrial de los ácidos grasos provenientes del metabolismo de los triglicéridos y el aumento de citoquinas inflamatorias son considerados factores causales de inflamación, fibrosis y daño hepático. Algunos autores consideran a la EHGNA como un componente del síndrome metabólico; ambos están estrechamente asociados con elevados niveles de insulina (27).

Los mecanismos patogénicos exactos de la EHGNA no son completamente conocidos. No se ha documentado con certeza por qué en algunos pacientes la esteatosis simple evoluciona más lentamente a la esteatohepatitis; mientras que en otros, se presenta con un progreso más rápido a estadios más avanzados. Lo cierto es que existe un limitado conocimiento de la historia natural debido a la ausencia de estudios de seguimiento prospectivos clínicos e histológicos a largo plazo (28).

\section{FACTORES DE RIESGO Y COMPLICACIONES}

El EHGNA se asocia con factores de riesgo tales como: la obesidad, la hipertrigliceridemia, la quimioterapia, la alimentación parenteral, los corticoides, la tolerancia a la glucosa alterada, y la diabetes mellitus. Las últimas condiciones han sido asociadas con la forma más severa de la enfermedad y a un pronóstico más desfavorable $(27,29)$. La asociación de la EHGNA con el índice de masa corporal elevado, es de aproximadamente el $58 \%$ en los individuos con sobrepeso y el $90 \%$ con obesidad mórbida. De todas maneras, no está claro si la infiltración grasa precede al desarrollo de las alteraciones del metabolismo de la glucosa y de los lípidos 0 se desarrolla de manera concomitante. La asociación de EHGNA con otras enfermedades, se ha demostrado que en personas con diabetes mellitus tipo 2 la prevalencia se eleva al $70 \%$ y en aquellos con dislipidemia puede alcanzar el $80 \%$ $(27,30,31)$.

La esteatohepatitis no alcohólica se asocia con el riesgo aumentado de cáncer hepático y la muerte por enfermedades cardiovasculares relacionadas con las causas hepáticas (32). La esteatosis simple puede seguir en sus inicios un curso caracterizado por una progresión lenta, no obstante, algunos estudios han demostrado que 30 a $40 \%$ de pacientes que sufren de EHGNA están en riesgo de desarrollar fibrosis y una cirrosis potencial (33).

Los pacientes con diagnóstico de EHGNA presentan un incremento del riesgo de padecer afecciones hepáticas crónicas, enfermedad cardiovascular y diabetes mellitus, lo que explica la mortalidad aumentada comparada con la población no enferma (34). En pacientes con EHGNA se ha reportado una incidencia de hasta $50 \%$ en el desarrollo de fibrosis hepática, $15 \%$ de ellos evolucionan a la cirrosis y un $3 \%$ a insuficiencia hepática. La probabilidad de 
padecer carcinoma hepatocelular es de un $7 \%$ en un periodo de 10 años $(35,36)$.

\section{HÍGADO GRASO NO ALCOHÓLICO Y DIABETES MELLITUS TIPO 2}

La diabetes mellitus tipo 2 y la esteatosis hepática no alcohólica son factores de riesgo recíprocos. La prevalencia de enfermedad hepática grasa no alcohólica en los pacientes diabéticos tipo 2 es aproximadamente de 50 a $70 \%$ (37). Existe una proporción significativa de pacientes con EHGNA que desarrollan a su vez síndrome metabólico años después del diagnóstico de la enfermedad, caracterizado por obesidad, diabetes mellitus tipo 2, dislipidemia e hipertensión $(29,38,31)$.

En sujetos con EHGNA, el hígado es resistente a la insulina, lo que lleva a la sobreproducción de glucosa y de lipoproteínas de muy baja densidad (VLDL). La glucosa, a su vez, estimula la secreción de insulina, lo que induce hiperinsulinemia. La presencia de glucosa alterada en ayuno (GAA) es un factor de riesgo importante para el desarrollo de DMT2 y en pacientes con EHGNA se ha demostrado mayor riesgo de desarrollo de GAA, con un cociente de riesgo ajustado (aHR) 1.823 (IC 95\% 1.316-2.534, $\mathrm{p}<0.001)$ para 46 hombres $y$ de 2.016 (IC 95\% 1.117-3.6, $p=0.02$ ) en mujeres $(1,39)$.

\section{DIAGNÓSTICO}

Continúa representando en la actualidad un reto tanto el diagnóstico como en el tratamiento médico del EHGNA. Su desarrollo generalmente asintomático hace complicado establecer un diagnóstico oportuno. Sólo los pacientes con EHGNA avanzada suelen referir cierto grado de malestar general, molestias en el hipocondrio derecho, astenia u otro síntoma digestivo, durante la exploración física con menos frecuencia es detectable hepatomegalia. En la mayoría de los casos el diagnóstico de EHGNA se debe al hallazgo de algunas alteraciones en marcadores serológicos de funcionamiento hepático (40). La biopsia del hígado ha sido considerada por años como la prueba de oro 0 criterio de verdad para el diagnóstico de la fibrosis hepática, y es el único método fiable para diferenciar la esteatosis simple de la fibrosis en los pacientes con EHGNA y descartar la presencia de otras enfermedades hepáticas causales o concomitantes (41). Sin embargo, el uso de las biopsias hepáticas has sido un tanto relegada en la última década por ser pobre su fiabilidad para graduar la fibrosis pues solo se toma muestra de un área limitada del hígado; por otra parte, es una prueba invasiva, no exenta de complicaciones, y por consiguiente inapropiada para el seguimiento de pacientes con EHGNA, después de la intervención terapéutica (42). Desde el punto de vista histopatológico el EHGNA comprende una serie de lesiones: solo esteatosis, esteatosis con inflamación lobular o portal sin balonización y esteatosis con balonización, pero sin inflamación. El diagnóstico de EHGNA requiere la presencia conjunta de esteatosis, balonización e inflamación lobular. Otras características histológicas que pueden observarse aun cuando no son necesarias para el diagnóstico son: inflamación portal, infiltrados 
polimorfonucleares, cuerpos de MalloryDenk, cuerpos apoptósicos, núcleos con vacuolas claras, esteatosis microvacuolar y megamitocondrias (27).

\section{TRATAMIENTO}

El manejo de los pacientes con EHGNA consiste en tratar la enfermedad hepática y las comorbilidades metabólicas tales como obesidad, hiperlipidemias y diabetes mellitus tipo 2. Una combinación de una dieta hipocalórica (reducción diaria de 500-1000 kcal) y ejercicio de intensidad moderada es probable que genere pérdida de peso corporal con el tiempo y reducción de la esteatosis hepática (15). La pérdida de peso corporal de al menos 3\% $-5 \%$ del peso corporal parece necesaria para mejorar la esteatosis, pero se necesita un mayor descenso de peso (7\% -10\%) para mejorar la mayoría de las características histopatológicas incluida la fibrosis hepática. Estas modificaciones en el estilo de vida mejoran también los niveles de transaminasas; sin embargo, cuando se pierde más del $10 \%$ del peso corporal, el $90 \%$ de los pacientes han logrado resolución completa de la esteatohepatitis $(1,15)$.

Los estudios por imágenes (ecografía abdominal, tomografía computarizada, resonancia magnética y elastografía) son técnicas no invasivas que permiten la evaluación cuali-cuantitativa de la grasa hepática $(27,43)$. La ecografía abdominal es una de las técnicas imagenológicas más utilizadas para detectar pacientes con aumento de la ecogenicidad hepática. Algunos estudios han demostrado que tiene entre $60-94 \%$ de sensibilidad, y de un 66-95 \% de especificidad para el diagnóstico de la esteatosis hepática (44). Muchos autores consideran la ecografía en modo B convencional es una herramienta simple, extensamente disponible y de primera línea para identificar el grado de infiltración grasa del hígado en los pacientes con sospecha de EHGNA, aun cuando es una técnica operador dependiente y de naturaleza cualitativa (17).

\section{RESULTADOS}

Los diabéticos tipo 2 con EHGNA incluidos en este estudio, presentaron edades ( $n=33 ; r=38-74$ ) y media de 54,9 $\pm 9,1$ años, con predominio del sexo masculino ( $\mathrm{n}=20 ; 60,6 \%$ ) (TABLA 1). Se determinaron varios grados de lesión hepática por ecografía abdominal; el aumento moderado de la ecogenicidad aportó el mayor número de casos $(n=15$; 45,5\%) (Ver FIGURA 1).

\begin{tabular}{|l|c|c|c|c|c|c|}
\hline \multicolumn{1}{|c|}{ TABLA 1. Distribución por edad y sexo. } \\
Hospital "H. Ameijeiras". La Habana. 2018 \\
\hline Edad & Fem & Porc. & Masc & Porc & Total & Porc \\
\hline $38-43$ & 4 & 12,12 & 2 & 6,06 & 6 & 18,18 \\
\hline $44-50$ & 3 & 9,09 & 2 & 6,06 & 5 & 15,15 \\
\hline $51-56$ & 1 & 3,03 & 6 & 18,18 & 7 & 21,21 \\
\hline $57-62$ & 3 & 9,09 & 5 & 15,15 & 8 & 24,24 \\
\hline $63-69$ & 2 & 6,06 & 5 & 15,15 & 7 & 21,21 \\
\hline Total & 13 & 39,39 & 20 & 60,61 & 33 & 100 \\
\hline $\begin{array}{l}|c| \\
\text { Fuente: Elaboración propia con información de la planilla } \\
\text { de datos }\end{array}$ \\
\hline
\end{tabular}

Los marcadores serológicos indirectos más alterados fueron la glicemia $(n=29$; $87,8 \%)$, los triglicéridos $(n=25 ; 75,8 \%)$ y el colesterol total ( $n=22 ; 66,7 \%)$ respectivamente. 
FIGURA 1. Imágenes de ecográficas de lesiones por esteatosis hepática en pacientes con EHGNH por Diabetes Metitus Tipo

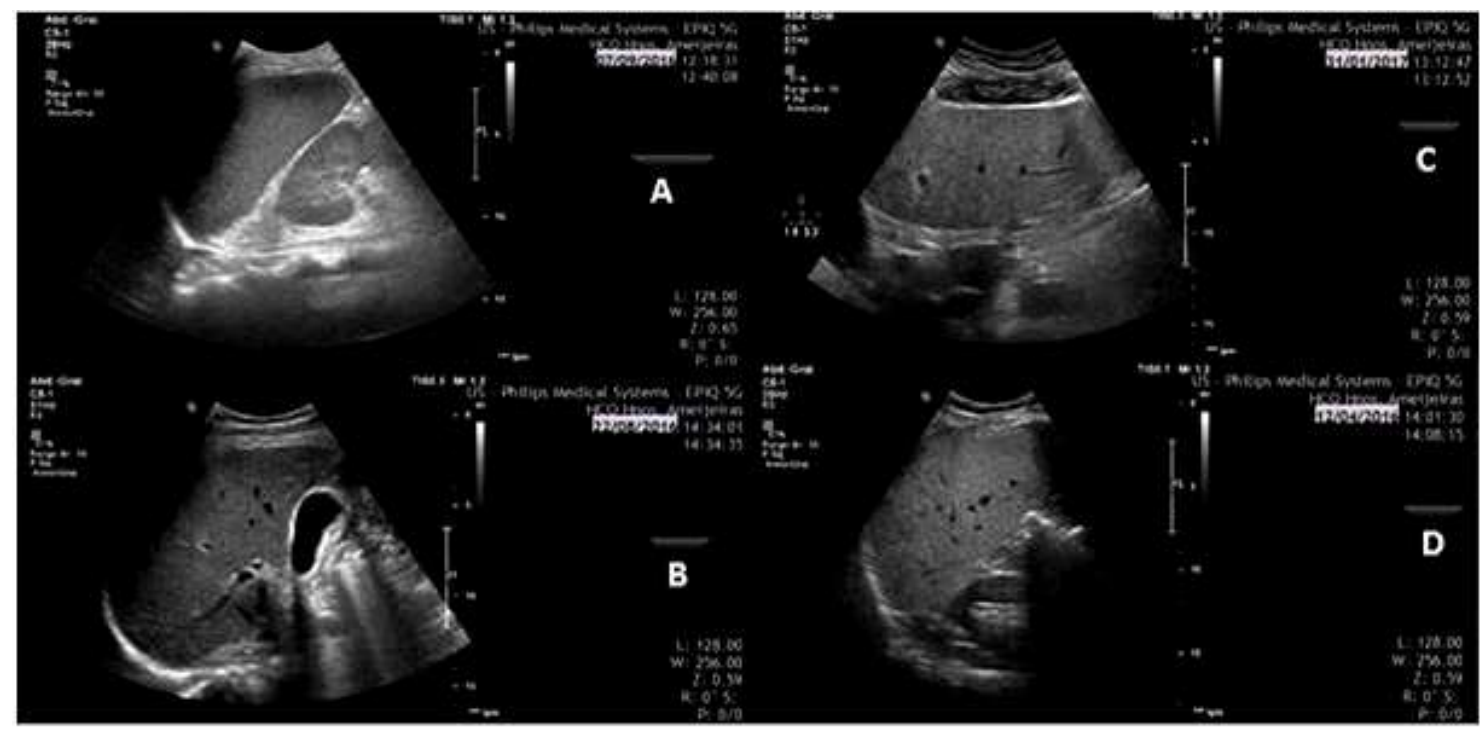

* (Casos A y B): Esteatosis Hepática Ligera. (Casos C y D): Esteatosis Hepática Moderada

Fuente: Cortesía del Departamento de Imagenología, Hospital “Hermanos Ameijeiras". La Habana, Cuba; 2018.

TABLA 2. Distribución de variables humorales y el grado de ecogenicidad hepática en diabéticos tipo 2 con EHGNA. Hospital "H. Ameijeiras". La Habana. 2018

\begin{tabular}{|c|c|c|c|c|c|c|c|c|}
\hline \multirow{3}{*}{$\begin{array}{l}\text { Variables } \\
\text { Humorales }\end{array}$} & \multicolumn{6}{|c|}{ Grados de ecogenicidad } & \multicolumn{2}{|c|}{ Prueba Kolmogorov } \\
\hline & \multicolumn{2}{|c|}{$\begin{array}{l}\text { Leve } \\
(n=10)\end{array}$} & \multicolumn{2}{|c|}{ Moderado $(n=15)$} & \multicolumn{2}{|c|}{$\begin{array}{c}\text { Severo } \\
(n=8)\end{array}$} & \multirow{2}{*}{$\begin{array}{c}\mathrm{T} \\
\text { Student }\end{array}$} & $p$ \\
\hline & Media & $\mathrm{DE}$ & Media & $\mathrm{DE}$ & Media & $\mathrm{DE}$ & & $<0,0001$ \\
\hline Glicemia & 6,81 & 1,03 & 7,12 & 1,60 & 11,16 & 1,90 & 0,99 & $<0,0001$ \\
\hline Triglicéridos & 2,22 & 0,56 & 2,53 & 0,74 & 3,50 & 0,85 & 0,82 & $<0,0001$ \\
\hline Colesterol & 5,11 & 1,85 & 5,60 & 1,03 & 6,99 & 1,11 & $\overline{0,94}$ & $<0,0001$ \\
\hline ALAT & 33,50 & 16,53 & 42,87 & 10,95 & 53,38 & 9,26 & 1,00 & $<0,0001$ \\
\hline ASAT & 31,50 & 18,67 & 43,20 & 14,80 & 61,38 & 14,81 & 1,00 & $<0,0001$ \\
\hline GGT & 27,80 & 14,71 & 43,00 & 14,14 & 51,75 & 20,50 & 1,00 & $<0,0001$ \\
\hline
\end{tabular}

Por su parte las aminotransferasas más afectadas fueron la alanino-transaminasa (ALAT) con ( $\mathrm{n}=21 ; 63,6 \%)$ (Ver FIGURA 2).

Los valores promedios de glicemia fueron de $(8,01 \pm 2,35 \mathrm{mmol} / \mathrm{L})$; triglicéridos $(2,67 \pm 0,86 \quad \mathrm{mmol} / \mathrm{L}) \quad y$ colesterol total $(5,79 \pm 1,49 \mathrm{mmol} / \mathrm{L})$. La totalidad de los casos ( $n=8 ; 100 \%)$ con aumento severo de la ecogenicidad concomitaron con alteraciones en los parámetros humorales: hiperglicemia
$(11,16 \pm 1,90 \mathrm{mmol} / \mathrm{L})$, hipercolesterolemia $(6,99 \pm 1,1 \mathrm{mmol} / \mathrm{L})$ e hipertrigliceridemia

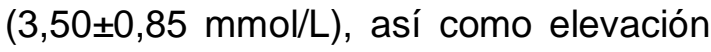
moderada de las transaminasas a predominio de la ASAT $(61,38 \pm 14,81 \mathrm{U} / \mathrm{I})$. La prueba de bondad de ajuste de Kolmogorov y la $\mathrm{T}$ de Student muestran distribución de normalidad para cada una de las variables y los grados de lesión diagnosticadas por ecografía (Ver TABLA 2; FIGURA 3).

e264 Revista Médica Sinergia Vol. 4 (8), Agosto 2019 - ISSN:2215-4523 / e-ISSN:2215-5279 
FIGURA 2. Determinaciones humorales en diabéticos tipo 2 con EHGNA. Hospital "H. Ameijeiras". La Habana. 2018

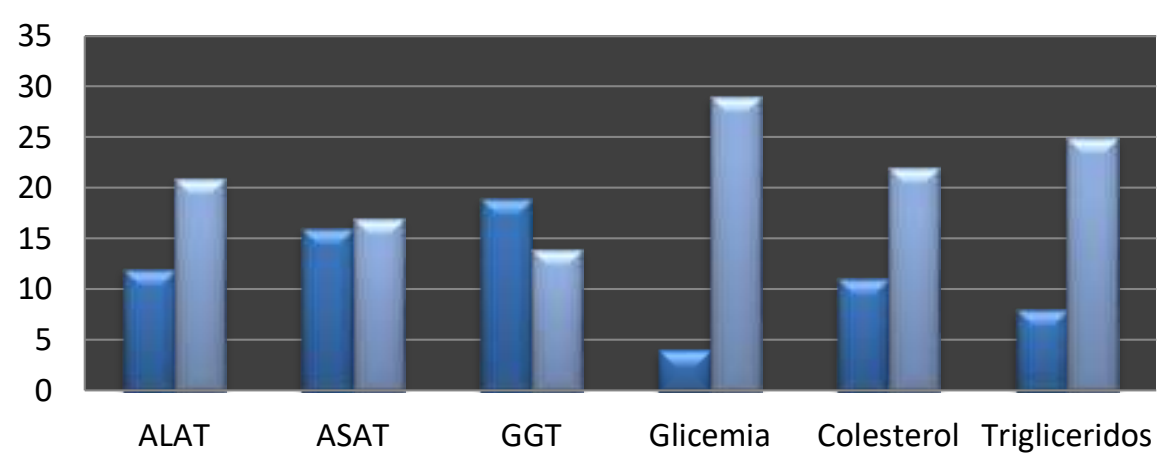

Normal

$\square$ Alterado

Fuente: Elaborada por los autores a partir de datos primarios

Figura 3. Distribución de variables humorales y el grado de ecogenicidad hepática en diabéticos tipo 2 con EHGNA. Hospital "H. Ameijeiras". La Habana. 2018

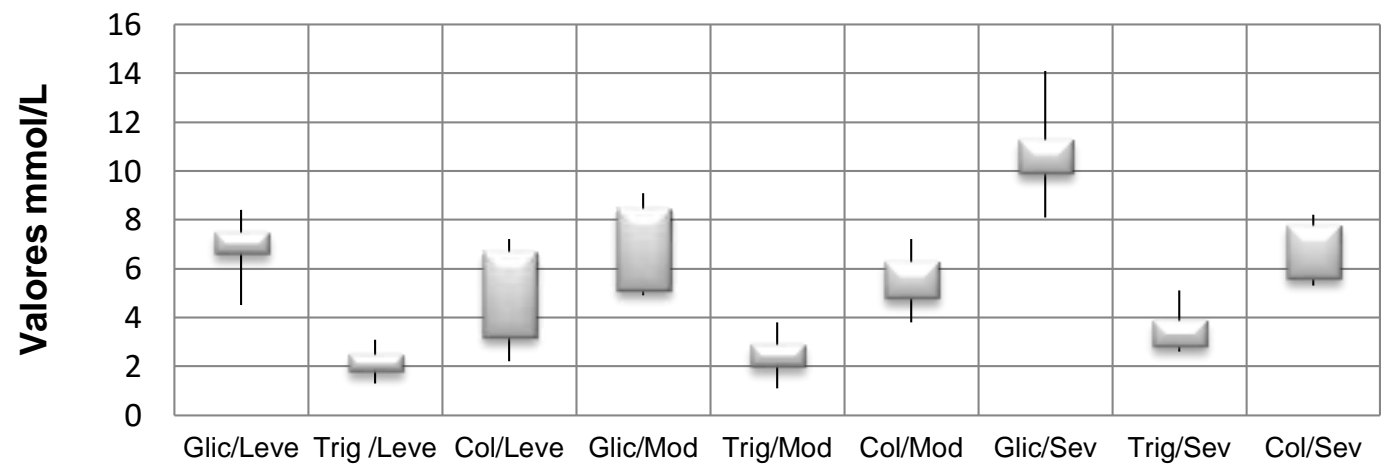

Fuente: Elaborada por los autores a partir de datos primarios

El desarrollo del EHGNA de los pacientes con diabetes mellitus tipo 2 evaluados en el presente estudio parece estar ligado a descompensación metabólica crónica.

Los niveles de glicemia y enzima ASAT ofrecieron la correlación estadística más significativa con los grados de lesión hepática diagnosticada por ecografía abdominal, siendo los valores de Rho Spearman de $r=0,65$ y $r=0,61$ respectivamente. Los análisis de varianza para todas las variables serológicas indirectas resultó inferior 0,05 (FIGURA 4).

\section{DISCUSIÓN}

Los datos obtenidos en esta investigación coinciden con los resultados de Friedrich Rust, et al. y Álvarez \& Anders quienes reportan una prevalencia de EHGNA en edades entre los 18 a 77 años, con un promedio de 55 años y predominio del sexo masculino $(45,46)$. 
FIGURA 4. Correlación entre marcadores serológicos y grados de ecogenicidad hepática en diabéticos tipo 2 con EHGNA. Hospital "Hermanos Ameijeiras". La Habana. 2018
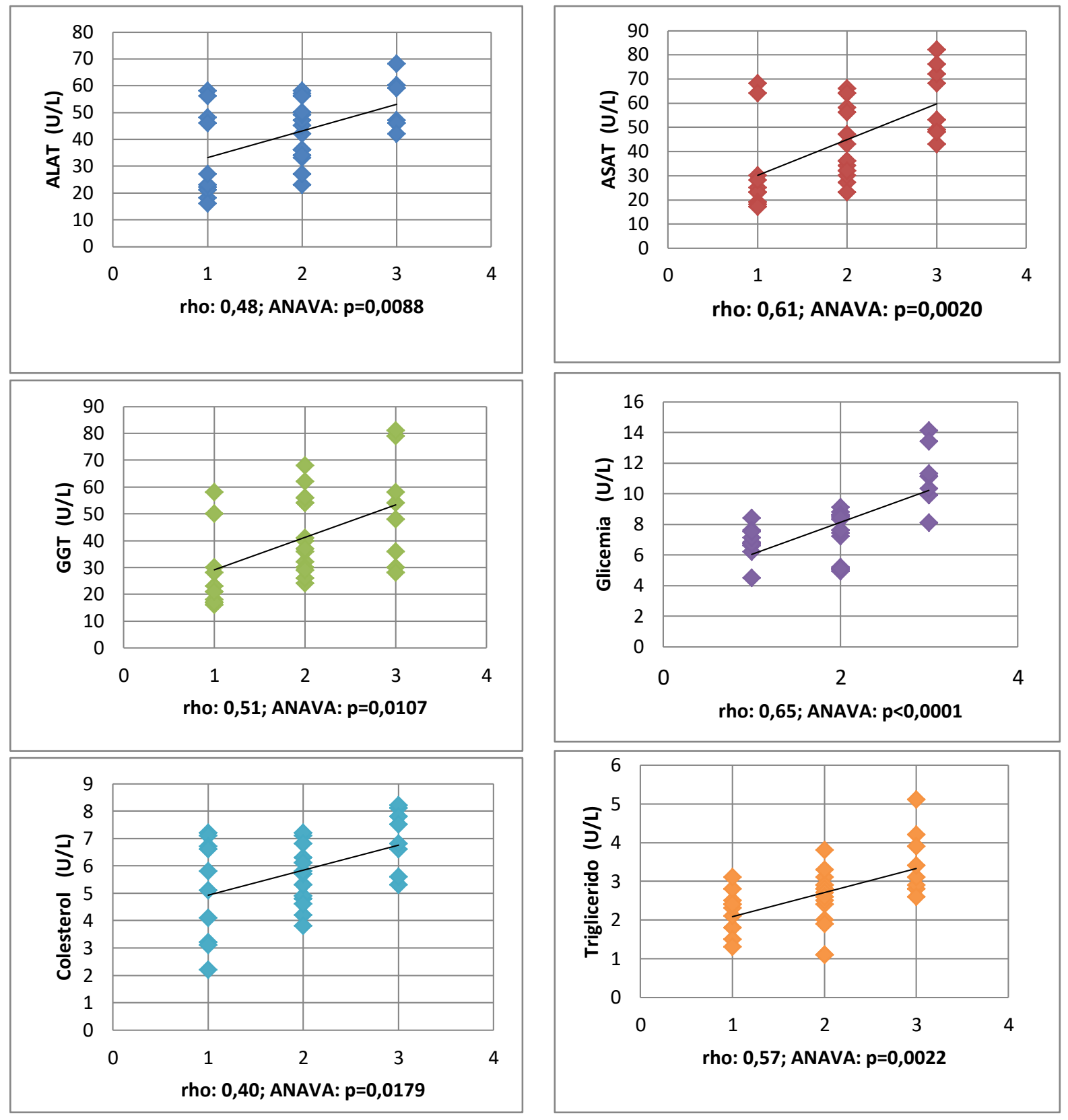

${ }^{*}$ Rho: Coeficiente de correlación de Spearman ${ }^{* *}$ ANAVA: Análisis de la varianza

Nota: Se operacionalizó 1, 2 y 3 a los grados de ecogenicidad Fuente: Elaborada por los autores a partir de datos primarios 
Por otra parte Yoneda, et al., consideran además la edad (> 45 años) como factor predictivo de fibrosis avanzada en pacientes con EHGNA (47). Loomba, et al., y Suárez González, et al., observaron que las mujeres padecían en un $68 \%$ la EHGNA, a diferencia del presente estudio donde aventajan los pacientes del sexo masculinos $(28,48)$. Sin embargo, varios autores justifican la diferencia de género, y abogan por una mayor prevalencia de la EHGNA en hombres $(49,1)$.

Según Singh \& Muir y Webb, et al., la ecografía es la modalidad de elección para determinar la esteatosis hepática, sin dudar que su resultado es más cualitativo, menos sensible y más dependiente del operador $(50,51)$. Los marcadores ecográficos obtenidos en la presente investigación discrepan a los mostrados por Harshit \& Aggarwal, quienes identificaron mediante ecografía una hiperecogenicidad moderada en un $56,5 \%$, leve en $23,4 \%$ y severa en 20,1 $\%$ de los casos (52). Por su parte Cassinotto, et al., refirío que la hiperecogenicidad hepática leve se ostenta en sólo un 37,4 \% y severidad moderada en un $32 \%$; datos que distan del presente análisis (53). Sin embargo, De Lédinghen \& Wong y Lee \& Park no avalaron lo hasta ahora señalado; estos últimos autores, refieren que la mayoría de los pacientes muestran en la ecografía un grado leve de hiperecogenicidad $61,8 \%$, moderado en $25 \%$ y solo un $5,26 \%$ aumento severo de la ecogenicidad hepática; los datos demostrados por estos investigadores si concuerdan con la presente investigación $(54,55)$.

En la literatura se reconoce que la elevación de las transaminasas, en especial la (ALAT) aumenta las posibilidades de presentar EHGNA. Se ha descrito además que en los pacientes portadores de EHGNA, se encuentra elevación moderada de aminotransferasas ALAT y ASAT, hipercolesterolemia, hipertrigliceridemia e hiperglicemia. No obstante otros autores como Cequera \& García de León exponen que aunque la ALAT es un test útil, no es válido para predecir la presencia de EHGNA dado que la enfermedad puede cursar con ALAT normal (56). Según Yilmaz y Suárez González, et al., la hiperglicemia y la hiperlipidemia (fundamentalmente la hipertrigliceridemia), son hallazgos frecuentes en los pacientes con EHGNA, asociados generalmente en el curso de un síndrome metabólico (28,57). Los anteriores planteamientos fueron corroborados en el análisis de la actual investigación.

\section{CONCLUSIONES}

En pacientes con diabetes mellitus tipo 2 el hígado graso no alcohólico prevaleció en hombre, mayores de 45 años con hiperecogenicidad moderada, patrón hepático difuso y aumento moderado de aminotransferasas ASAT y ALAT. La hiperglicemia, hipertrigliceridemia e hipercolesterolemia fueron proporcionales al daño mostrado por los marcadores ecográficos. El desarrollo del hígado graso no alcohólico pareciera estar ligado a descompensación metabólica crónica e insulinorresistencia. Los niveles de glicemia y enzima ASAT fueron los marcadores serológicos indirectos que presentaron la correlación estadística más significativa con los 
grados de lesión hepática diagnosticada por ecografía abdominal.

Se recomienda el uso de modelos predictivos a partir de variables clínicas, marcadores serológicos indirectos y ecográficos en el diagnóstico y seguimiento a pacientes con EHGNA por su sencillez, y buena correlación con las técnicas más complejas. Realizar una segunda fase de la investigación con uso de técnica elastográfica para reevaluar el objetivo del presente estudio. La estratificación de riesgo con el empleo de dichos herramientas permitiría implementar medidas tempranas para reducir la morbimortalidad por causa cardiovascular.

\section{RESPONSABILIDADES ÉTICAS}

Protección a personas y animales: Los autores declaran que para esta investigación no se han realizado ningún proceder experimental en seres humanos ni en animales.

Confidencialidad de los datos. Los autores declaran que en este artículo se manejó de manera confidencial los datos clínicos de todos los pacientes.

Derecho a la privacidad y consentimiento informado. Los autores declaran que en este artículo no aparecen datos de pacientes.

\section{AGRADECIMIENTOS}

Se agradece la tutoría del Dr. C. Miguel Ángel Rodríguez Allende; a las consultas realizadas a los profesores Dr. Orlando Valls, Lic. Jorge Luis Hernández y Dr. Francisco Fernández Valdés.

\section{REFERENCIAS}

1. Velarde-Ruiz Velasco J, Mercado-Jáuregui L, Bocaletti-García M, Pinzón-Flores O, Barrientos-Ávalos J. Enfermedad hepática por hígado graso no alcohólico ¿Dónde estamos y hacia dónde vamos? Revista Médica MD. 2019; 10(2): p. 155-163.

2. Hatziagelaki E, Karageorgopoulos DE, Chounta A, Tsiavou A, Falagas ME, Dimitriadis G. Predictors of Impaired Glucose Regulation in Patients with Non-Alcoholic Fatty Liver Disease. Experimental Diabetes Research. 2012; 2012: p. 1-7. https://doi.org/10.1155/2012/351974

3. Bonapace S, Valbusa F, Bertolini L, Pichiri I, Mantovani A, Rossi A, Zenari L, Barbieri E, Targher G. Nonalcoholic Fatty Liver Disease Is Associated with Aortic Valve Sclerosis in Patients with Type 2 Diabetes Mellitus. Sookoian SC. PLoS ONE. $2014 \quad 02 \quad$ 05; 9(2): e88371. https://doi.org/10.1371/journal.pone.0088371

4. Lonardo A, Sookoian S, Pirola CJ, Targher G. Non-alcoholic fatty liver disease and risk of cardiovascular disease. Metabolism. $2016 \quad$ 08; $65(8)$ : p. 1136-1150. https://doi.org/10.1016/j.metabol.2015.09.017

5. Wanless IR, Lentz JS. Fatty liver hepatitis (steatohepatitis) and obesity: An autopsy study with analysis of risk factors. Hepatology. 1990 Nov; $12(5)$ :1106-1110. https://doi.org/10.1002/hep.1840120505

6. Lizardi-Cervera J, Becerra Laparra I, Chávez-Tapia NC, Ramos Ostos ME, Uribe Esquivel M. Prevalencia de hígado graso no alcohólico y síndrome metabólico en población asintomática. Revista Gastroenterología Mexicana. 2006; 71(4): p. 453-459 
7. Longo DL, Kasper DL, Jameson JL, Fauci AS, Hauser SL, Loscalzo J. HARRISON. PRINCIPIOS DE MEDICINA INTERNA. 18th ed.: McGRAW-HILL INTERAMERICANA; 2012

8. Younossi ZM, Koenig AB, Abdelatif D, Fazel Y, Henry L, Wymer M. Global epidemiology of nonalcoholic fatty liver disease-Meta-analytic assessment of prevalence, incidence, and outcomes. Hepatology. 201602 22; 64(1): p. 73-84. https://doi.org/10.1002/hep.28431

9. Younossi ZM, Stepanova M, Afendy M, Fang Y, Younossi Y, Mir H, Srishord M. Changes in the Prevalence of the Most Common Causes of Chronic Liver Diseases in the United States From 1988 to 2008. Clinical Gastroenterology and Hepatology. 2011 06; 9(6): 524-530.e1. https://doi.org/10.1016/j.cgh.2011.03.020

10. Diehl AM, Day C. Cause, Pathogenesis, and Treatment of Nonalcoholic Steatohepatitis. Longo DL. New England Journal of Medicine. 2017 Nov 23; 377(21): p. 2063-2072. https://doi.org/10.1056/neimra1503519

11. Ministerio de Salud Pública. Anuario Estadístico de Salud. La Habana:, Dirección de registros médicos y estadísticas de Salud; 2018. Aprox. 193 p

12. Santiesteban Rodríguez M. Ultrasonido y Tomografía Axial Computarizada en el diagnóstico de la Esteatosis Hepática no Alcohólica. Tesis de Terminacion de Especialidad. La Habana: Hospinal Nacional "Hermanos Ameijeiras", Imagenología; 2013

13. Elvírez G, Castellanos F, Santillán L, Dorta G, Galbán G, Arús S. Liver Stiffness Reference Values for Healthy Cuban Adults. MEDICC Review. 2018 January ; 20(1): p. 24-28

14. EASL-EASD-EASO Clinical Practice Guidelines for the management of non-alcoholic fatty liver $\begin{array}{llllll}\text { disease. Journal of } 2016 \quad \text { Hepatology. } & \text { 06; } & \text { 64(6): } & \text { p.1388-1402. }\end{array}$ https://doi.org/10.1016/j.jhep.2015.11.004

15. Chalasani N, Younossi Z, Lavine JE, Diehl AM, Brunt EM, Cusi K, Charlton M, Sanyal AJ. The diagnosis and management of non-alcoholic fatty liver disease: Practice Guideline by the American Association for the Study of Liver Diseases, American College of Gastroenterology, and the American Gastroenterological Association. Hepatology. 201205 29; 55(6): p. 2005-2023. https://doi.org/10.1002/hep.25762

16. Caballería L, Saló J, Berzigotti A, Planas R, Vila C, Huertas C, Torres M, Torán P, Caballería J. Hígado graso no alcohólico. Documento de posicionamiento de la Societat Catalana de Digestologia. $\begin{array}{llllll}\text { Gastroenterología } \quad \text { y } \quad \text { Hepatología. } 2014 \quad 06 ; & 37(6): & \text { p.372-383. }\end{array}$ https://doi.org/10.1016/i.gastrohep.2014.03.005

17. Khov N. Bedside ultrasound in the diagnosis of nonalcoholic fatty liver disease. World Journal of Gastroenterology. 2014; 20(22): 6821. https://doi.org/10.3748/wjg.v20.i22.6821

18. Lee TH, Han† SH, Yang JD, Kim $\ddagger$ D, §, Ahmed\| M. Prediction of Advanced Fibrosis in Nonalcoholic Fatty Liver Disease: An Enhanced Model of BARD Score. Gut and Liver. 201305 15; 7(3): p.323-328. https://doi.org/10.5009/gnl.2013.7.3.323

19. Goulart AC, Oliveira IRSD, Alencar AP, Santos MSCD, Santos IS, Martines BMR, Meireles DP, Martines JADS, Misciagna G, Benseñor IM, Lotufo PA. Diagnostic accuracy of a noninvasive hepatic ultrasound score for non-alcoholic fatty liver disease (NAFLD) in the Brazilian Longitudinal Study of Adult Health (ELSA-Brasil). Sao Paulo Medical Journal. 2015 04; 133(2): p. 115-124. https://doi.org/10.1590/1516-3180.2014.9150812

20. Machado MV. Non-alcoholic fatty liver disease: What the clinician needs to know. World Journal of Gastroenterology. 2014; 20(36): 12956. https://doi.org/10.3748/wjg.v20.i36.12956 
21. Alkhouri N, McCullough AJ. Noninvasive Diagnosis of NASH and Liver Fibrosis Within the Spectrum of NAFLD. Gastroenterol Hepatol. 2012 Oct; 8(10): p.661-668

22. Kumar R, Rastogi A, Sharma MK, Bhatia V, Tyagi P, Sharma P, Garg H, Chandan Kumar KN, Bihari C, Sarin SK. Liver Stiffness Measurements in Patients with Different Stages of Nonalcoholic Fatty Liver Disease: Diagnostic Performance and Clinicopathological Correlation. Digestive Diseases and Sciences. 201207 12; 58(1): p.265-274. https://doi.org/10.1007/s10620012-2306-1

23. Satapathy S, Sanyal A. Epidemiology and Natural History of Nonalcoholic Fatty Liver Disease. Seminars in Liver Disease. 201509 17; 35(03): p.221-235. https://doi.org/10.1055/s-00351562943

24. Lindenmeyer CC, McCullough AJ. The Natural History of Nonalcoholic Fatty Liver Disease-An Evolving View. Clinics in Liver Disease. 2018 02; 22(1): 11-21. https://doi.org/10.1016/i.cld.2017.08.003

25. Williams CD, Stengel J, Asike MI, Torres DM, Shaw J, Contreras M, Landt CL, Harrison SA. Prevalence of Nonalcoholic Fatty Liver Disease and Nonalcoholic Steatohepatitis Among a Largely Middle-Aged Population Utilizing Ultrasound and Liver Biopsy: A Prospective Study. Gastroenterology. 2011 01; 140(1): 124-131. https://doi.org/10.1053/j.gastro.2010.09.038

26. Sun B, Li Y, Lan Y. Fibroscan for diagnosis and treatment of chronic liver disease. World Chinese Journal of Digestology. 2015; 23(9): p.1433. https://doi.org/10.11569/wcjd.v23.i9.1433

27. Graffigna M, Catoira N, Soutelo J, Azpelicueta A, Berg G, Perel C, Migliano ME, Aranguren M, Musso C, Farias J. Diagnóstico de esteatosis hepática por métodos clínicos, bioquímicos y por imágenes. Revista Argentina de Endocrinología y Metabolismo. 2017 01; 54(1): 37-46. https://doi.org/10.1016/i.raem.2016.12.001

28. Suárez González M, López Mejia VM, Eirin Rey JM, González González EL, Medina Garrido Y, Díaz Oliva SE. Enfermedad hepática grasa no alcohólica. Algunas consideraciones diagnósticas. Acta Médica del Centro. 2017; 11(2): p. Aprox. 9.

29. Hui E, Xu A, Bo Yang H, Lam KSL. Obesity as the common soil of non-alcoholic fatty liver disease and diabetes: Role of adipokines. Journal of Diabetes Investigation. 201305 06; 4(5): p.413-425. https://doi.org/10.1111/jdi.12093

30. Ahmed M. Non-alcoholic fatty liver disease in 2015. World Journal of Hepatology. 2015; 7(11): p.1450. https://doi.org/10.4254/wih.v7.i11.1450

31. Zhang Q, Lu L. Nonalcoholic fatty liver disease: dyslipidemia, risk for cardiovascular complications, and treatment strategy. J Clin Transl Hepatol. 2015; 3: p. 78-84. Disponible en: http://www.ncbi.nlm.nih.gov/pubmed/26357637

32. Fruci B, Giuliano S, Mazza A, Malaguarnera R, Belfiore A. Non-alcoholic fatty liver: a possible new target for type 2 diabetes prevention and treatment. International Journal of Molecular Sciences. 2013; 14: p. 22933-22966. https://doi.org/10.3390/ijms141122933

33. Leite N, Villela Nogueira C, Cardoso C, Salles G. Non-alcoholic fatty liver disease and diabetes: from physiopathological interplay to diagnosis and treatment. World Journal of Gastroenterol. 2014; 20(26): p. 8377-8392. https://doi.org/10.3748/wjg.v20.i26.8377

34. Athyros V, Tziomalos K, Katsiki N, Doumas M, Karagiannis A, Mikhailidis D. Cardiovascular risk across the histological spectrum and the clinical manifestations of non-alcoholic fatty liver disease: an update. World Journal of Gastroenterology. 2015; 21(22): p. 6820-6834.

https://doi.org/10.3748/wjg.v21.i22.6820 
35. Arab J, Candia R, Zapata R, Muñoz C, Arancibia J, Poniachik J. Management of non-alcoholic fatty liver disease: An evidence-based clinical practice review. World Journal of Gastroenterol. 2014; 20(34): p. 12182-12201. https://doi.org/10.3748/wjg.v20.i34.12182

36. Buzzeti E, Lombardi R, De Luca L, Tsochatzis E. Non-invasive assessment of fibrosis in patients with non-alcoholic fatty liver disease. International Journal of Endocrinology. 2015; 34(28): p. 1 9. https://doi.org/10.1155/2015/343828

37. S B, Valbusa F, Bertolini L, Pichiri I, Montovani A, Rossi A, et al. Non-alcoholic fatty liver disease is associated with aortic valve sclerosis in patients with type 2 Diabetes Mellitus. Plos one. 2014 February; 9(2): p. 1 -6. https://doi.org/10.1371/journal.pone.0088371

38. Armstrong M, Hazlehurst J, Parker R, Koushiappi E, Mann J, Sheeba K, et al. Severe asymptomatic non-alcoholic fatty liver disease in routine diabetes care; a multi-disciplinary team approach to diagnosis and management. Q Journal Medical. 2014; 107: p. 33-41. https://doi.org/10.1093/qimed/hct198

39. Lllalukka S, Yki-Jarvinen $\mathrm{H}$. Non-alcoholic fatty liver disease and risk of Type 2 diabetes. Best Practice and Research Clinical Endocrinology and Metabolism. 2016;: p. 1-11. https://doi.org/10.1016/j.beem.2016.06.006

40. Villarejo Botija M, Prieto Zancudo C, Martín Fuertes L. Evaluación de la fibrosis en la esteatosis hepática no alcohólica con fibroscán. med gen y fam. 2015; 4(1): p. 25-29. https://doi.org/10.1016/j.mgyf.2015.05.007

41. Spengler E, Loomba R. Recommendations for diagnosis, referral for liver biopsy, and treatment of nonalcoholic fatty liver disease and nonalcoholic steatohepatitis. Mayo Clinic Proceedings. 2015;: p. 1233-1246. Disponible en: http://refhub.elsevier.com/S0326-4610(16)300651/sbref0555. https://doi.org/10.1016/i.mayocp.2015.06.013

42. Le S, Park S. Radiologic evaluation of non-alcoholic fatty liver disease. World Journal of Gastroenterol.. 2014; 20(23): p. 7392-73402. https://doi.org/10.3748/wjg.v20.i23.7392

43. Singh D, Das C, Baruah M. Imaging of non alcoholic fatty liver disease: A road less travelled. Indian J Endocrinol Metab. 2013; 17: p. 990-995. Disponible en: http://wwwpubmedcentral.nih.gov/articlerender.fcgi?artid=3872717\&tool=pmcentrez\&rendertype= Abstract. https://doi.org/10.4103/2230-8210.122606

44. Csendes P, Paolinelli P, Busel D, Venturelli V, Rodríguez J. Hígado graso: ultrasonido y correlación anátomo-patológica. Rev Chil Radiol. 2004; 10(2): p. 50-52. https://doi.org/10.4067/S0717-93082004000200003

45. Friedrich Rust M, Poynard T, Castera L. Critical comparison of elastography methods to assess chronic liver disease. Rev Gastroenterol Hepatol. 2016; 13(7): p. 402-411. https://doi.org/10.1038/nrgastro.2016.86

46. Álvarez D, Anders M. Utilidad de la Elastografía de transición (Fibroscan) en la evaluación de la fibrosis hepática en pacientes con hepatopatía crónica. Rev Med Bue Air. 2012; 72(1): p. 10-14

47. Yoneda M, Suzuki K, Kato S, Fujita K, Nozaki Y, Hosono K, et al. Nonalcoholic fatty liver disease: US-based acoustic radiation force impulse elastography.. Radiol. 2010; 256: p. 640-647. https://doi.org/10.1148/radiol.10091662

48. Loomba R, Abraham M, Tech B, etal. Association between diabetes, family history of diabetes and risk of nonalcoholic steatohepatitis and fibrosis. Hepatology. 2012; 56(3): p. 943-951. https://doi.org/10.1002/hep.25772 
Correlación entre marcadores serológicos y ecográficos en pacientes con hígado graso no alcohólico y diabetes mellitus tipo 2

Dra. Naibel Quevedo Ramírez; Dr. Raydel Pérez Castillo; Dra. Isnielly Virgen Sánchez Gendriz

49. Williams C, Stengel J, Asike M, etal. Prevalence of nonalcoholic fatty liver disease and nonalcoholic steatohepatitis among a largely middle- aged population utilizing ultrasound and liver biopsy: a prospective study. Gastroenterology. 2011; 140: p. 124-131. https://doi.org/10.1053/j.gastro.2010.09.038

50. Singh S, Muir A. American Gastroenterological Association Institute Technical review on the role of Elastography in chronic liver diseases. Gastroenterol. 2017; 152(6): p. 1544-1577.

https://doi.org/10.1053/j.gastro.2017.03.016

51. Webb M, Yeshua H, Zelber Sagi S, Santo E, Brazowski E, Halpern Z, et al. Diagnostic value of a computerized hepatorenal index for sonographic quantification of liver steatosis. Am J Roentgenol. 2009; 192: p. 909-914.Disponible en: http://refhub.elsevier.com/S03264610(16)30065-1/sbref0495.

https://doi.org/10.2214/AJR.07.4016

52. Harshit G, Aggarwal S. Utility of Transient Elastography (FibroScan) and impact of bariatric surgery on non-alcoholic fatty liver disease (NAFLD) in morbidly obese patients. Surg obes relat dis. $2017 ; 9(5)$.

53. Cassinotto C, Boursier J, de Ledinghen V. Liver stiffness in non-alcoholic fatty liver disease: a comparison of supersonic shear imaging, FibroScan,and ARFI with liver biopsy. Hepatol. 2016; 63(6): p. 550-557. https://doi.org/10.1002/hep.28394

54. De Lédinghen V, Wong G. Controlled attenuation parameter for the diagnosis of steatosis in nonalcoholic fatty liver disease. Jour Gastroenterol. 2016; 31(4): p. 848-855.

https://doi.org/10.1111/igh.13219

55. Lee H, Park S. Discrimination of non-alcoholic steatohepatitis using Transient Elastography in patients with non-alcoholic fatty liver disease. Plos One. 2016; 11(6): p. 157-358.

56. Cequera A, García de León Méndez M. Biomarcadores para fibrosis hepática, avances, ventajas y desventajas. Rev Gastroenterol Méx. 2014; 79(3): p. 187-199.

https://doi.org/10.1016/j.rgmx.2014.05.003

57. Yilmaz Y. NAFLD in the absence of metabolic syndrome: different epidemiology, pathogenetic mechanisms, risk factors for disease progression? Semin Liver Dis. 2014; 32(1): p. 14-21. Disponible

en: https://www.researchgate.net/publication/221896525 NAFLD in the Absence of Metabolic Sy ndrome Different Epidemiology Pathogenetic Mechanisms Risk Factors for Disease Progre ssion. 